PSS PROCEEDINGS

\title{
Event Generators
}

\section{Leif Lönnblad*}

Department of Astronomy and Theoretical Physics, Lund University

E-mail: leif.lonnbladethep.lu.se

A brief overview of the current state of general purpose event generators, focusing on recent developments in extending the precision of QCD to next-to-leading order.

XXII. International Workshop on Deep-Inelastic Scattering and Related Subjects, 28 April - 2 May 2014

Warsaw, Poland

${ }^{*}$ Speaker. 


\section{Introduction}

The importance of Monte Carlo event generators in high energy physics has increased tremendously as the complexity of experiments and analysis has developed during the last couple of decades. Today, no one would dream of designing a new collider or detector without extensive studies using event generators, nor would any analysis be made without detailed checking of predictions from event generators to get a detailed understanding of the physics and systematics of the observables studied.

Up until recently, however, the standard event generators have been lacking somewhat in precision. All current general purpose event generators ${ }^{1}$ basically implement the same structure for generating fully exclusive events (see [4] for a detailed review of the physics of general purpose event generators):

1. Pick a hard sub-process and generate its kinematics according to a fixed order matrix element and a given PDF parameterization.

2. Allow incoming and outgoing legs to generate bremsstrahlung according to a parton shower model, effectively resumming large logarithms from soft and collinear poles.

3. Include additional parton-parton scatterings according to some model to get a reasonable description of the underlying event.

4. Hadronize all outgoing partons together with the beam remnants according to some model to obtain a hadronic final state.

\section{Decay unstable hadrons.}

In each of these steps, it is easy to identify precision problems. The matrix elements used in (1) are typically calculated to leading order (LO) and only include a very limited number of external legs. The parton showers in (2) will at best resum emissions to next-to-leading logarithmic (NLL) precision, but often only the leading logarithms are correct beyond the leading colour $\left(N_{c} \rightarrow \infty\right)$ approximation. Furthermore, the models for hadronization and multi-parton interactions ( 3 and 4 ) inherently depend on non-perturbative assumptions, the precision of which are typically beyond formal control.

Furthermore, the parton shower resummation is done in the framework of collinear factorization, and it is clear that with the large energy at LHC one would assume that small- $x$ effects should become important and the need for $k_{\perp}$-factorization apparent.

In the following I will discuss briefly recent developments to increase the precision of event generators and raise some questions concerning the small- $x$ issue.

\section{The NLO revolution}

The last decade has seen formidable progress in the calculation of matrix elements with several external legs to next-to-leading order (NLO) in $\mathrm{QCD}^{2}$. There now even exist programs where such

\footnotetext{
${ }^{1}$ i.e. Herwig++ [1], PYTHIA [2] and SHERPA [3].

${ }^{2}$ I cannot possible do justice to all results of these intricate calculations, and here limit the references to the more conceptual papers [5-14], which made this progress possible.
} 
matrix elements can be generated automatically, the most used one being MadGraph5_aMC@NLO [15].

The problem with these calculations has been to properly combine them with parton shower programs to obtain a reasonable description of the fully hadronic final states, but recently there has been great progress also in this area.

The main difficulties in combining matrix elements and parton showers has been the fact that both include terms in the expansion of $\alpha_{S}$ and it is crucial to avoid double counting. For the distribution of one extra jet from the parton shower it is sometimes possible to make a correction of the splitting functions used in the shower [16] and although attempts have been made to generalize this to higher jet multiplicities and even to NLO [17,18], no working implementation exists yet for LHC.

For multiple hard jets there instead exist a number of schemes for combining tree-level matrix elements with different multiplicities. The problem here is that since matrix element calculations in general are inclusive (gives at least $n$ jets), it is not possible to blindly add different jet multiplicities together. This was first solved in the so-called CKKW(-L) algorithms [19] ( [20]), by reweighting the matrix element states with the no-emission probabilities a parton shower would use, to obtain exclusive event. The algorithms also involves a merging scale to regularize the divergencies in the matrix elements, and a procedure to ensure that only the matrix element populate the phase space above this scale and that the parton shower populates the full phase space below. These and similar schemes (e.g. [21] and [22]) suffers from a small dependence on the merging scale which, although it formally is below the precision of the shower, makes it difficult to take the scale too low. ${ }^{3}$

To obtain increased precision we need to go to beyond tree-level matrix elements. However, this means we have to be even more careful when adding parton showers. There are two basic methods for this, MC@NLO [24] and POWHEG [25]. Symbolically one can describe an NLO calculation for an $n$-jet observable as follows

$$
\langle\mathscr{O}\rangle_{\mathrm{NLO}}=\int d \phi_{n}\left(B_{n}+V_{n}\right) \mathscr{O}_{n}+\int d \phi_{n+1} B_{n+1} \mathscr{O}_{n+1},
$$

where $B_{n}$ is the LO matrix element, $V_{n}$ is the virtual contribution and $B_{n+1}$ is the real emission contribution. To avoid the fact that both the real emission and virtual contributions are both infinite, the standard technique is to introduce a subtraction functions $S_{n+1}$ with the same divergencies as the real emission contribution, but allows for analytical integration to cancel the divergences of the virtual terms,

$$
\langle\mathscr{O}\rangle_{\mathrm{NLO}}=\int d \phi_{n}\left(B_{n}+V_{n}+\int d \phi_{+1} S_{n+1}\right) \mathscr{O}_{n}+\int d \phi_{n+1}\left(B_{n+1}-S_{n+1}\right) \mathscr{O}_{n+1} .
$$

The idea behind NC@ NLO is based on the fact that parton shower splitting functions exactly cancel the divergencies in the desired way, and using these one can then simply generate the two samples (with $n$ and $n+1$ jets) and add the parton shower to each and then add the samples together without any double counting of terms.

\footnotetext{
${ }^{3}$ In a later development of CKKW-L, the dependence on the merging scale can be almost completely removed using a technique described in [23].
} 
The POWHEG strategy corresponds to calculating a K-factor for each $n$-jet phase space point, symbolically

$$
\bar{B}_{n}=B_{n}+V_{n}+\int d \phi_{+1} B_{n+1},
$$

and then generating the first emission using the ratio $B_{n+1} / B_{n}$ rather than the standard parton shower splitting functions (similarly to what was done in [16]) and then continuing with the normal parton shower.

MC@NLO and POWHEG are equivalent to NLO precision, but differ in higher orders since contrary to MC@NLO, in POWHEG the emission of a second parton is multiplied with the NLO K-factor.

It is important to realize that when generating events with these procedures, not all observables measured on these events will come out correct to NLO. As an example, if we generate the process $p p \rightarrow Z^{0}$ to NLO, any observable that depends on one extra jet will only be correct to LO. Hence, the rapidity of the $Z^{0}$ is correct to NLO, while the transverse momentum is only correct to LO, since this transverse momentum must be balanced by a jet. To get the transverse momentum distribution of $Z^{0}$ correct to NLO, we therefore need to start with the NLO matrix element for $Z^{0}+1$-jet. But then the problem is that the NLO corrections become unstable for small transverse momenta, requiring a resummation of large logarithms in addition, to obtain sensible results.

This problem has now been solved in the latest developments in the field, and there is now several available schemes for combining NLO event samples for several multiplicities into one run so that predictions for any observable supported by one of the NLO calculations will come out correct to NLO. The first method is based on CKKW-L and was outlined in [26] and first implemented for LHC processes in SHERPA [27, 28]. It involves a careful expansion of the noemission probabilities in the parton shower, removing the terms which are already included in any of the NLO calculation used.

As for the tree-level CKKW-L algorithms mentioned above, there is a dependence on the merging scale used to separate the parton shower and matrix element regions of phase space, and in order to not destroy the precision of one jet multiplicity when adding a NLO calculation for a higher multiplicity, it is important not to take this merging scale too low. This problem is solved in the so-called UNLOPS scheme ${ }^{4}[29,30]$ where higher multiplicity event samples are both added as is, and subtracted from lower multiplicity samples by reclustering emissions.

Two other algorithms for multi-jet NLO matching should be mentioned. The so-called FxFx scheme [31] is similar to the ones above but is based on the MLM scheme for tree-level merging [21]. This also has a merging scale dependence, but it is not clear how large it is. The final technique is called MiNLO [32] and is based on an analytic resummation of the one-jet NLO calculation ensuring that also the zero-jet NLO cross section is correct. This method has no merging scale at all, but it is unclear how it can be extended to higher multiplicities.

\section{The small- $x$ conundrum}

At HERA, one of the most striking results was the sharp rise of the gluon distribution at small $x$. Although this could be described by standard NLO DGLAP evolution, there were several

\footnotetext{
4 available in PYTHIA8 and soon also in Herwig++.
} 
final state observables, such as forward energy flows and forward jets (see e.g. [33]), which could not be described by DGLAP-based parton shower generators, hinting at the break-down of collinear factorization. Naturally there has been some efforts to see similar effects at the LHC.

The situation at LHC is, however, somewhat different. To get really small $x$-values one needs to go to really small transverse momenta of jets, and then one has to worry about the underlying event. An observable sensitive to interesting small- $x$ effects would typically involve looking at correlations between jets at large rapidity distances, but here DGLAP-based generators such as PYTHIA8 would produce several multiple (semi-)hard scatterings in the same collisions as described in [34], possibly hiding effects of small- $x$ resummation effects. Also the other general purpose (DGLAP-based) generators implements similar multi-parton (MPI) interaction models.

To avoid effects of MPI one can try to go to higher jet transverse momenta, as was done by CMS in [35] where they studied the ratio of inclusive to exclusive two-jet cross sections as a function of rapidity separation - an observable explicitly designed to reveal BFKL-like evolution. The comparison with event generators turned out to be quite surprising. The data is well described by the DGLAP-based PYTHIA generator, while two programs which explicitly include small- $x$ resummation, CASCADE [36] and HEJ [37], failes miserably.

There are, of course, also other programs that simulates small $x$ resummation, such as the Regge-based approaches, PHOJET [38], the SHRiMPS model in the SHERPA generator, and EPOS [39] (which also includes an interesting hydro-dynamical evolution phase) and also the DIPSY program [40] based on initial-state dipole evolution in impact parameter space, but all of these lack the precision needed at high transverse momenta.

The jury is still out, but it is clear that there is a lot of work still to be done to understand properly possible effects of small- $x$ resummation at the LHC.

\section{Conclusions}

The event generators have now entered the precision era. In a near future the state-of-the-art will be to make predictions for any observable to NLO precision, using multi-jet NLO matching. Before too long we can also expect to see proper NNLO predictions (see e.g. [41] and [32]).

Also, a lot of progress has been made in improving generators for minimum bias physics and multi-parton interactions. But here we inevitably enter the non-perturbative domain, where formal precisions is hard to obtain.

\section{References}

[1] M. Bahr, S. Gieseke, M. Gigg, D. Grellscheid, K. Hamilton, et al., Herwig++ Physics and Manual, Eur.Phys.J. C58 (2008) 639-707, arXiv:0803.0883 [hep-ph] .

[2] T. Sjostrand, S. Mrenna, and P. Z. Skands, A Brief Introduction to PYTHIA 8.1, Comput.Phys.Commun. 178 (2008) 852-867, arXiv:0710.3820 [hep-ph] .

[3] T. Gleisberg, S. Hoeche, F. Krauss, M. Schonherr, S. Schumann, et al., Event generation with SHERPA 1.1, JHEP 0902 (2009) 007, arXiv: 0811.4622 [hep-ph] .

[4] A. Buckley, J. Butterworth, S. Gieseke, D. Grellscheid, S. Hoche, et al., General-purpose event generators for LHC physics, Phys.Rept. 504 (2011) 145-233, arXiv:1101.2599 [hep-ph] . 
[5] Z. Bern, L. J. Dixon, D. C. Dunbar, and D. A. Kosower, One loop n point gauge theory amplitudes, unitarity and collinear limits, Nucl.Phys. B425 (1994) 217-260, arXiv : hep-ph/9403226 [hep-ph].

[6] R. Britto, F. Cachazo, and B. Feng, Generalized unitarity and one-loop amplitudes in N=4 super-Yang-Mills, Nucl.Phys. B725 (2005) 275-305, arXiv: hep-th/0412103 [hep-th] .

[7] A. Denner and S. Dittmaier, Reduction schemes for one-loop tensor integrals, Nucl.Phys. B734 (2006) 62-115, arXiv: hep-ph/0509141 [hep-ph] .

[8] G. Ossola, C. G. Papadopoulos, and R. Pittau, Reducing full one-loop amplitudes to scalar integrals at the integrand level, Nucl.Phys. B763 (2007) 147-169, arXiv: hep-ph/0609007 [hep-ph] .

[9] R. K. Ellis, W. Giele, and Z. Kunszt, A Numerical Unitarity Formalism for Evaluating One-Loop Amplitudes, JHEP $\mathbf{0 8 0 3}$ (2008) 003, arXiv: 0708 . 2398 [hep-ph ] .

[10] G. Ossola, C. G. Papadopoulos, and R. Pittau, On the Rational Terms of the one-loop amplitudes, JHEP 0805 (2008) 004, arXiv: 0802.1876 [hep-ph] .

[11] R. K. Ellis, W. T. Giele, Z. Kunszt, and K. Melnikov, Masses, fermions and generalized D-dimensional unitarity, Nucl.Phys. B822 (2009) 270-282, arXiv:0806.3467 [hep-ph] .

[12] C. Berger, Z. Bern, L. Dixon, F. Febres Cordero, D. Forde, et al., An Automated Implementation of On-Shell Methods for One-Loop Amplitudes, Phys.Rev. D78 (2008) 036003, arXiv: 0803.4180 [hep-ph].

[13] S. Becker, C. Reuschle, and S. Weinzierl, Numerical NLO QCD calculations, JHEP 1012 (2010) 013, arXiv:1010.4187 [hep-ph].

[14] F. Cascioli, P. Maierhofer, and S. Pozzorini, Scattering Amplitudes with Open Loops, Phys.Rev.Lett. 108 (2012) 111601, arXiv:1111.5206 [hep-ph] .

[15] J. Alwall, R. Frederix, S. Frixione, V. Hirschi, F. Maltoni, et al., The automated computation of tree-level and next-to-leading order differential cross sections, and their matching to parton shower simulations, arXiv:1405.0301 [hep-ph].

[16] M. Bengtsson and T. Sjostrand, A Comparative Study of Coherent and Noncoherent Parton Shower Evolution, Nucl.Phys. B289 (1987) 810.

[17] W. Giele, D. Kosower, and P. Skands, Higher-Order Corrections to Timelike Jets, Phys.Rev. D84 (2011) 054003, arXiv:1102.2126 [hep-ph].

[18] L. Hartgring, E. Laenen, and P. Skands, Antenna Showers with One-Loop Matrix Elements, JHEP 1310 (2013) 127, arXiv:1303.4974 [hep-ph].

[19] S. Catani, F. Krauss, R. Kuhn, and B. Webber, QCD matrix elements + parton showers, JHEP 0111 (2001) 063, arXiv:hep-ph/0109231 [hep-ph].

[20] L. Lonnblad, Correcting the color dipole cascade model with fixed order matrix elements, JHEP 0205 (2002) 046, arXiv:hep-ph/0112284 [hep-ph].

[21] M. L. Mangano, M. Moretti, and R. Pittau, Multijet matrix elements and shower evolution in hadronic collisions: $W b \bar{b}+n$ jets as a case study, Nucl.Phys. B632 (2002) 343-362, arXiv:hep-ph/0108069 [hep-ph].

[22] S. Mrenna and P. Richardson, Matching matrix elements and parton showers with HERWIG and PYTHIA, JHEP 0405 (2004) 040, arXiv : hep-ph/ 0312274 [hep-ph ] . 
[23] L. Lonnblad and S. Prestel, Unitarising Matrix Element + Parton Shower merging, JHEP 1302 (2013) 094, arXiv: 1211.4827 [hep-ph].

[24] S. Frixione and B. R. Webber, Matching NLO QCD computations and parton shower simulations, JHEP 0206 (2002) 029, arXiv : hep-ph/ 0204244 [hep-ph ] .

[25] P. Nason, A New method for combining NLO QCD with shower Monte Carlo algorithms, JHEP 0411 (2004) 040, arXiv:hep-ph/0409146 [hep-ph].

[26] N. Lavesson and L. Lonnblad, Extending CKKW-merging to One-Loop Matrix Elements, JHEP 0812 (2008) 070, arXiv:0811.2912 [hep-ph] .

[27] T. Gehrmann, S. Hoche, F. Krauss, M. Schonherr, and F. Siegert, NLO QCD matrix elements + parton showers in $e^{+} e^{-} \rightarrow$ hadrons, JHEP 1301 (2013) 144, arXiv:1207.5031 [hep-ph] .

[28] S. Hoeche, F. Krauss, M. Schonherr, and F. Siegert, QCD matrix elements + parton showers: The NLO case, JHEP 1304 (2013) 027, arXiv: $1207.5030 \quad$ [hep-ph] .

[29] L. Lönnblad and S. Prestel, Merging Multi-leg NLO Matrix Elements with Parton Showers, JHEP 1303 (2013) 166, arXiv:1211.7278 [hep-ph] .

[30] S. Plätzer, Controlling inclusive cross sections in parton shower + matrix element merging, JHEP 1308 (2013) 114, arXiv:1211.5467 [hep-ph] .

[31] R. Frederix and S. Frixione, Merging meets matching in MC@NLO, JHEP 1212 (2012) 061, arXiv:1209.6215 [hep-ph].

[32] K. Hamilton, P. Nason, C. Oleari, and G. Zanderighi, Merging H/W/Z $+O$ and 1 jet at NLO with no merging scale: a path to parton shower + NNLO matching, JHEP 1305 (2013) 082, arXiv: 1212.4504.

[33] H1 Collaboration Collaboration, C. Adloff et al., Forward jet and particle production at HERA, Nucl.Phys. B538 (1999) 3-22, arXiv: hep-ex/9809028 [hep-ex] .

[34] T. Sjostrand and M. van Zijl, A Multiple Interaction Model for the Event Structure in Hadron Collisions, Phys.Rev. D36 (1987) 2019.

[35] CMS Collaboration Collaboration, S. Chatrchyan et al., Ratios of dijet production cross sections as a function of the absolute difference in rapidity between jets in proton-proton collisions at $\sqrt{s}=7$ TeV, Eur.Phys.J. C72 (2012) 2216, arXiv:1204.0696 [hep-ex] .

[36] H. Jung, S. Baranov, M. Deak, A. Grebenyuk, F. Hautmann, et al., The CCFM Monte Carlo generator CASCADE version 2.2.03, Eur.Phys.J. C70 (2010) 1237-1249, arXiv:1008.0152 [hep-ph] .

[37] J. R. Andersen and J. M. Smillie, Multiple Jets at the LHC with High Energy Jets, JHEP 1106 (2011) 010, arXiv:1101.5394 [hep-ph].

[38] R. Engel, J. Ranft, and S. Roesler, Hard diffraction in hadron hadron interactions and in photoproduction, Phys.Rev. D52 (1995) 1459-1468, arXiv: hep-ph/9502319 [hep-ph] .

[39] T. Pierog, I. Karpenko, J. Katzy, E. Yatsenko, and K. Werner, EPOS LHC : test of collective hadronization with LHC data, arXiv:1306.0121 [hep-ph] .

[40] C. Flensburg, G. Gustafson, and L. Lonnblad, Inclusive and Exclusive Observables from Dipoles in High Energy Collisions, JHEP 1108 (2011) 103, arXiv:1103.4321 [hep-ph] .

[41] S. Hoeche, Y. Li, and S. Prestel, Drell-Yan lepton pair production at NNLO QCD with parton showers, arXiv:1405.3607 [hep-ph]. 